\title{
E1 retorno de las Migraciones Circulares: La regulación de las migraciones profesionales
}

\author{
Antonio Alaminos \\ Cristina López \\ Begoña López \\ Ignacia Perea \\ Oscar Santacreu
}

Instituto de Desarrollo Social y Paz

\section{Resumen}

Nuevamente, reaparece la noción de "migraciones circulares" como procedimiento de racionalización de las migraciones con motivación laboral. Es un concepto que produce tanto reacciones adversas como favorables entre los investigadores. Como tantos propuestas, sus virtudes y defectos dependerán más de cómo se concrete su reglamentación y aplicación práctica que de sus contenidos y potencialidades. Una parte sustantiva de las migraciones corresponde con motivaciones socioeconómicas, asociadas a la movilidad laboral. Para este tipo de migración, la ruptura con los elementos culturales, de vida cotidiana, y con las redes sociales de su país de origen son consecuencias no deseadas de la movilidad. La contribución más visible de su esfuerzo, en las sociedades de origen, son las remesas económicas. Las migraciones circulares proponen que la movilidad incorpore la noción de regreso. Es decir, que la migración suponga un momento de su ciclo vital, donde la restitución al entorno social de origen pueda ser una realidad. Incluyendo las ventajas para una nueva migración posterior. Las consecuencias de esta reestructuración en el horizonte vital de los migrados son importantes. Permite reintegrar a las sociedades de origen capital humano formado y cualificado, con las consecuencias positivas para el desarrollo social y económico en estas sociedades. Mantiene la calidad de vida afectiva y emocional en los migrantes y su entorno familiar. Intensifica la relación entre las dos sociedades y simplifica los problemas que supone la convivencia entre sistemas culturales o religiosos contradictorios. Especialmente referidos a la libertad e igualdad. Son múltiples los ejemplos referidos a la violencia de género, sobre niños, etc., inaceptables en los estados sociales de derecho democráticos que predominan en las sociedades occidentales.

Ciertamente el ámbito de aplicación óptimo lo define la migración económica. Este enfoque migratorio excluye otro tipo de migración especialmente importante: la no económica, donde la migración viene motivada por buscar nuevos horizontes vitales, explorar estilos de vida alternativos, persecución política, etc.

\begin{abstract}
Again, the notion of "circular migration" as a process of rationalization of migrations with job motivation is reappearing. It is a concept that produces both favourable and adverse reactions among researchers. Like so many proposals, their strengths and weaknesses depend more on how is realized its regulation and its practical application than on their content and potential. A substantial part of migration corresponds to socio-economic reasons, associated with job mobility. For this type of migration, the break with the cultural
\end{abstract}


elements of everyday life and the social networks from their home country are unintended consequences of mobility. The most visible contribution of their efforts in the countries of origin is the economic remittances. Circular migration suggests that mobility incorporates the notion of return. In other words, that migration involves a moment of their life cycle, where the return to their social environment of origin can become a reality, including benefits for a new migration later. The consequences of this restructuring on the horizon of life of the migrants are important. It allows reintegrate skilled human capital to the societies of origin, with positive consequences for social and economic development in these societies. It maintains the quality of emotional life for the migrants and their families. It intensifies the relationship between the societies and simplifies the problems of coexistence of contradictory religious or cultural systems, with particular reference to freedom and equality. There are numerous examples relating to gender violence, children, etc, that are unacceptable in the democratic Western societies.

Certainly the optimal scope is defined by economic migration. This migratory approach excludes other types of migration especially important: the Non-economic migration, with other motivations as for example to look for new horizons of life, exploring alternative lifestyles, political persecution, and so on.

\section{El contexto migratorio}

La migración y la movilidad de las personas, especialmente aquellas altamente cualificadas ${ }^{1}$ y el capital humano, en general, son procesos en continuo crecimiento desde los inicios de la globalización. Se trata de fenómenos arraigados en una dinámica histórica en la cual la transferencia de conocimientos y habilidades constituyen un elemento fundamental en la economía mundial. Sin embargo, ha sido en las últimas dos décadas, a raíz del rápido desarrollo e implantación de las tecnologías de la información y la comunicación y la aceleración de la globalización que ello conlleva, cuando se le ha concedido mayor importancia en los espacio de discusión y un papel relevante en las investigaciones internacionales.

La creciente demanda en los países desarrollados de esta categoría profesional conformada por personas de alta cualificación, no queda al margen de controversias respecto a su posible impacto negativo en el desarrollo económico en general, así como de los recursos humanos en particular, de los países en desarrollo; a pesar de la función de la movilidad en la integración de estos países en la economía mundial. Es innegable que la movilidad de las personas de alta cualificación tiene un impacto diferente sobre cada región, reportando beneficios también distintos para cada una de ellas. La movilidad de las personas vinculadas con el savoir-faire del mundo académico y técnico se ha equiparado a la "internacionalización del conocimiento" y la "circulación de ideas y de los portadores de ideas" (Bourdieu, 1991; en Sabour, 2001).

\footnotetext{
1 "Cualificado" se considera a menudo en términos de logros educativos (véase Lowell y Findlay, 2001:3). "La movilidad de las personas altamente cualificadas" se refiere al movimiento de las personas con grados de educación superior, específicamente aquéllas con al menos cuatro años de educación tras el ciclo de primaria y secundaria (12 años). Movilidad significa cualquier tipo de movimiento internacional desde cualquier punto de partida, con patrones recurrentes o permanentes (ibid: 7 ).
} 
El aumento de la migración y la movilidad está relacionado con la nueva sociedad del conocimiento y de la economía, reflejando la persistente asimetría entre los países y regiones en el mundo. Así, la movilidad del capital humano en los países de la OCDE adopta el modelo de "circulación de cerebros"2 asociado, en gran parte, a migrantes temporales altamente cualificados; mientras que este mismo flujo toma, en los países en desarrollo en general, la forma de "fuga de cerebros", normalmente relacionada con el traslado permanente de la residencia de estos mismos migrantes (CEPES, 2004). Asimismo, esta situación se asienta sobre la desigual oferta de educación superior en cada región: mayor en los primeros países que en los segundos (Lowell, 2003).

Los factores de empuje/atracción son especialmente significativos para los casos de estudiantes, académicos e investigadores, ingenieros, profesionales de las tecnologías de la información, así como para profesionales de la medicina y directivos. Para éstos, un elemento importante a tener en cuenta en el aumento de esta movilidad es la búsqueda individual de mejores expectativas profesionales en los países desarrollados, donde se espera encontrar mejores condiciones de trabajo y remuneración, así como un clima propicio a la innovación y el desarrollo de la carrera profesional. Otro elemento a tener en consideración es la competencia que tiene lugar entre los países en su conjunto por atraer a trabajadores altamente cualificados para satisfacer las demandas de sus necesidades locales y las condiciones del mercado laboral. Asimismo, en el contexto actual, en el cual se enfatiza la innovación, para los países avanzados la educación y la mejora de la movilidad se convierten en componentes estratégicos del crecimiento económico. Easterlin (1981:2) destaca a este respecto que el crecimiento es una extensión o intensificación de la producción, mientras Landes (1980:111) afirma que las cuotas de crecimiento ascienden con la creación y utilización del conocimiento: "el corazón de todo el proceso de industrialización y desarrollo económico es intelectual: consiste en la adquisición y aplicación de un corpus de conocimiento sobre la técnica, es decir, las formas de hacer las cosas" (apud Smith: 7, en OCDE, 2001).

Considerando la opinión pública de las sociedades emisoras de emigración, las motivaciones más frecuentes están asociadas a la movilidad económica. Incluyendo la capacitación de capital humano, como es la "educación". Exclusivamente en sociedades con importantes conflictos toman relieve las motivaciones de "seguridad" o "refugio político".

\footnotetext{
2 "Circulación de cerebros" (intercambio): un país de origen puede intercambiar migrantes altamente cualificados con uno o varios países extranjeros. El intercambio se produce cuando la pérdida de los trabajadores nativos se compensa con un equivalente de entrada de trabajadores extranjeros altamente cualificados (ibid., 7).

3 "Fuga de cerebros" es cuando la emigración al extranjero, permanente o de larga duración, de personas altamente cualificadas, alcanza altos niveles sin ser equilibrados por los efectos "feedback" de las remesas, la transferencia de tecnología, las inversiones o el comercio. Disminuye el crecimiento económico a causa de la pérdida de rentabilidad de la inversión en la educación y el agotamiento de la fuente del activo capital humano del país. (ibíd., 7).
} 


\section{Tabla 1. Respecto a la gente que deja su país para vivir en otro lugar, cuál es la principal razón de su partida - trabajo, seguridad, educación, persecución política, matrimonio, o alguna otra razón?}

\begin{tabular}{|c|c|c|c|c|c|c|c|c|c|}
\hline & Jobs & Safety & Education & $\begin{array}{c}\text { Escape } \\
\text { political } \\
\text { persecution }\end{array}$ & $\begin{array}{l}\text { To get } \\
\text { married }\end{array}$ & Other & $\begin{array}{l}\text { Don't } \\
\text { know }\end{array}$ & Refused & Total \\
\hline Argentina & $85,50 \%$ & $8,60 \%$ & $0,60 \%$ & $0,30 \%$ & & $2,40 \%$ & $2,30 \%$ & $0,40 \%$ & $100,00 \%$ \\
\hline Bangladesh & $95,90 \%$ & $0,60 \%$ & $1,80 \%$ & $0,70 \%$ & & $0,40 \%$ & $0,60 \%$ & & $100,00 \%$ \\
\hline Bolivia & $96,00 \%$ & $1,00 \%$ & $0,60 \%$ & $0,50 \%$ & & $1,20 \%$ & $0,60 \%$ & $0,10 \%$ & $100,00 \%$ \\
\hline Brazil & $94,20 \%$ & $2,30 \%$ & $1,40 \%$ & & $0,10 \%$ & $0,70 \%$ & $1,20 \%$ & $0,10 \%$ & $100,00 \%$ \\
\hline Bulgaria & $90,20 \%$ & $5,00 \%$ & $2,60 \%$ & $0,20 \%$ & & $0,60 \%$ & $1,40 \%$ & & $100,00 \%$ \\
\hline $\begin{array}{l}\text { Chile } \\
\text { Czech }\end{array}$ & $85,90 \%$ & $3,00 \%$ & $6,10 \%$ & $1,30 \%$ & $0,40 \%$ & $2,00 \%$ & $1,40 \%$ & & $100,00 \%$ \\
\hline Republic & $59,60 \%$ & $0,90 \%$ & $5,10 \%$ & $15,60 \%$ & $1,30 \%$ & $13,80 \%$ & $2,00 \%$ & $1,80 \%$ & $100,00 \%$ \\
\hline Egypt & $42,60 \%$ & $9,70 \%$ & $21,60 \%$ & $8,00 \%$ & $14,30 \%$ & $2,80 \%$ & $1,00 \%$ & & $100,00 \%$ \\
\hline Ethiopia & $87,60 \%$ & $2,40 \%$ & $1,10 \%$ & $7,20 \%$ & & $0,10 \%$ & $1,00 \%$ & $0,60 \%$ & $100,00 \%$ \\
\hline Ghana & $90,20 \%$ & $0,60 \%$ & $7,20 \%$ & $0,30 \%$ & & $0,40 \%$ & $1,10 \%$ & $0,10 \%$ & $100,00 \%$ \\
\hline India & $82,50 \%$ & $3,40 \%$ & $11,80 \%$ & $0,50 \%$ & $0,20 \%$ & $0,10 \%$ & $1,30 \%$ & $0,00 \%$ & $100,00 \%$ \\
\hline Indonesia & $81,30 \%$ & $1,80 \%$ & $12,40 \%$ & $0,80 \%$ & $1,80 \%$ & & $1,90 \%$ & & $100,00 \%$ \\
\hline Ivory Coast & $95,20 \%$ & $0,80 \%$ & $2,70 \%$ & $0,60 \%$ & $0,30 \%$ & $0,30 \%$ & $0,10 \%$ & & $100,00 \%$ \\
\hline Jordan & $34,90 \%$ & $5,80 \%$ & $33,00 \%$ & $9,40 \%$ & $10,60 \%$ & $4,70 \%$ & $1,50 \%$ & $0,10 \%$ & $100,00 \%$ \\
\hline Kenya & $70,60 \%$ & $1,70 \%$ & $25,20 \%$ & $0,40 \%$ & $1,00 \%$ & $0,80 \%$ & $0,20 \%$ & $0,10 \%$ & $100,00 \%$ \\
\hline Kuwait & $24,40 \%$ & $3,00 \%$ & $26,80 \%$ & $7,00 \%$ & $3,40 \%$ & $7,60 \%$ & $27,00 \%$ & $0,80 \%$ & $100,00 \%$ \\
\hline Lebanon & $52,50 \%$ & $27,80 \%$ & $8,20 \%$ & $6,40 \%$ & $3,50 \%$ & $1,50 \%$ & $0,10 \%$ & & $100,00 \%$ \\
\hline Malaysia & $64,20 \%$ & $1,60 \%$ & $25,50 \%$ & $0,30 \%$ & $1,60 \%$ & $2,60 \%$ & $4,10 \%$ & $0,10 \%$ & $100,00 \%$ \\
\hline Mali & $92,00 \%$ & $0,40 \%$ & $2,00 \%$ & $0,60 \%$ & $0,70 \%$ & $4,00 \%$ & $0,30 \%$ & & $100,00 \%$ \\
\hline Mexico & $78,50 \%$ & $9,90 \%$ & $5,00 \%$ & $1,60 \%$ & $1,10 \%$ & $3,00 \%$ & $0,60 \%$ & $0,40 \%$ & $100,00 \%$ \\
\hline Morocco & $96,40 \%$ & $0,40 \%$ & $1,10 \%$ & $0,40 \%$ & $0,70 \%$ & $0,30 \%$ & $0,70 \%$ & & $100,00 \%$ \\
\hline Nigeria & $80,60 \%$ & $4,40 \%$ & $8,90 \%$ & $1,20 \%$ & $0,40 \%$ & $1,50 \%$ & $2,90 \%$ & $0,10 \%$ & $100,00 \%$ \\
\hline Pakistan & $76,80 \%$ & $2,40 \%$ & $5,20 \%$ & $0,20 \%$ & $0,70 \%$ & $2,50 \%$ & $11,50 \%$ & $0,70 \%$ & $100,00 \%$ \\
\hline \multicolumn{10}{|l|}{ Palestinian } \\
\hline Territories & $52,30 \%$ & $12,50 \%$ & $7,90 \%$ & $16,80 \%$ & $1,60 \%$ & $2,70 \%$ & $4,60 \%$ & $1,60 \%$ & $100,00 \%$ \\
\hline Peru & $95,10 \%$ & $0,80 \%$ & $1,00 \%$ & & $0,30 \%$ & $1,50 \%$ & $1,30 \%$ & $0,10 \%$ & $100,00 \%$ \\
\hline Poland & $95,60 \%$ & $1,20 \%$ & $1,80 \%$ & $0,20 \%$ & & $0,60 \%$ & $0,60 \%$ & & $100,00 \%$ \\
\hline Russia & $61,50 \%$ & $4,50 \%$ & $5,90 \%$ & $2,10 \%$ & $8,10 \%$ & $7,60 \%$ & $9,80 \%$ & $0,60 \%$ & $100,00 \%$ \\
\hline Senegal & $97,90 \%$ & $0,70 \%$ & $0,30 \%$ & & $0,40 \%$ & $0,60 \%$ & $0,10 \%$ & & $100,00 \%$ \\
\hline Slovakia & $94,40 \%$ & & $1,10 \%$ & $1,10 \%$ & $0,20 \%$ & $2,90 \%$ & $0,20 \%$ & & $100,00 \%$ \\
\hline South Africa & $76,10 \%$ & $14,50 \%$ & $4,80 \%$ & $1,40 \%$ & $0,20 \%$ & $1,80 \%$ & $1,20 \%$ & & $100,00 \%$ \\
\hline Tanzania & $68,60 \%$ & $1,80 \%$ & $16,20 \%$ & $0,30 \%$ & $0,30 \%$ & $6,40 \%$ & $6,30 \%$ & $0,10 \%$ & $100,00 \%$ \\
\hline Turkey & $79,90 \%$ & $2,60 \%$ & $3,60 \%$ & $2,10 \%$ & $0,40 \%$ & $0,80 \%$ & $7,60 \%$ & $3,00 \%$ & $100,00 \%$ \\
\hline Uganda & $83,20 \%$ & $2,80 \%$ & $6,60 \%$ & $2,90 \%$ & $0,50 \%$ & $1,20 \%$ & $2,80 \%$ & $0,10 \%$ & $100,00 \%$ \\
\hline Ukraine & $88,00 \%$ & $2,80 \%$ & $0,40 \%$ & $0,40 \%$ & $1,80 \%$ & $4,60 \%$ & $2,00 \%$ & & $100,00 \%$ \\
\hline Venezuela & $63,60 \%$ & $15,80 \%$ & $4,90 \%$ & $9,00 \%$ & $1,10 \%$ & $3,50 \%$ & $1,60 \%$ & $0,50 \%$ & $100,00 \%$ \\
\hline Total & $77,30 \%$ & $4,70 \%$ & $8,10 \%$ & $2,60 \%$ & $1,70 \%$ & $2,20 \%$ & $3,00 \%$ & $0,30 \%$ & $100,00 \%$ \\
\hline
\end{tabular}

Fuente: elaboración propia sobre microdatos encuesta PEW, 2007 
Este tipo de migración y movilidad (económica y educativa) ha supuesto históricamente costos y beneficios para los países de origen y los países de acogida, respectivamente, reafirmando la perspectiva de "pérdida de cerebros" y de capital humano cualificado que fluye fuera del país en busca de una mejor situación socioeconómica y el clima más conveniente para el rendimiento laboral. Sin embargo, si se incide en el regreso de los expatriados, en consonancia con la naturaleza de la migración circular, el impacto de esta migración puede ser valorada desde otro ángulo: el mantenimiento en sus países de origen de redes sociales y profesionales supone el fomento de la transferencia de conocimientos, las innovaciones y la capacidad tecnológica; además, la adquisición de mayor y distintiva experiencia y el aumento de la cualificación de este colectivo migrante al regresar a sus países de origen, propicia el establecimiento y fortalecimiento de redes de colaboración de expertos internacionales, para lo cual el empleo de la tecnología de la información, por ejemplo, resulta de gran utilidad.

Los puntos anteriormente mencionados son algunos de los impactos positivos indirectos (Lowell y Findlay, 2002; en Lowell, 2003) que estimulan la productividad y el desarrollo económico. Con todo ello, podríamos hablar aquí no tanto de "fuga de cerebros", como de "ganancia de cerebros" y "circulación de cerebros". Así pues resulta imperativo evaluar a través de la investigación comparativa este significativo, aunque complejo, feedback capaz de producir un crecimiento económico positivo global. A través de la promoción de estudios de estas características podremos obtener una imagen más precisa y detallada de los impactos a todos los niveles, sin obviar que la "fuga de cerebros" en los países en desarrollo resulta un gran desafío y los gobiernos de éstos han de focalizar sus esfuerzos para atraer de nuevo estas élites -proporcionando oportunidades para que estos migrantes de alta cualificación retornados puedan poner en práctica en sus países de origen su competencia, experiencia y capacidad-, ni a los expertos que afirman que los países desarrollados se benefician de la contribución de estas personas, como tampoco a las consideraciones de responsables políticos que apuntan a que todas las partes implicadas pueden beneficiarse de un régimen abierto (Straubhaar, 2000).

El contexto socio-económico y político y sus cambios, de los cuales los países del Sur han sido testigos, han alentado a una salida de estas personas altamente cualificadas hacia los países desarrollados. Las dimensiones políticas y socioeconómicas desempeñan un importante rol en esta movilidad hacia el espacio europeo y dentro de él. Los análisis sobre los efectos del nivel educativo en el comportamiento migratorio son muy amplios $y$, en líneas generales, sus conclusiones muestran que la educación aumenta la posibilidad de la movilidad, en especial la migración de trabajadores con niveles superiores de educación. Sin embargo, a nivel micro los estudios sobre las opciones de destino de estas personas y otros temas relacionados son mucho más escasos (Ritsilä, 2000: 2). Además, ha de considerarse que tanto la movilidad de este tipo de migración, como su integración en los países de acogida pueden diferir considerablemente de los patrones asociados a las experiencias de los inmigrantes menos cualificados. (Favell, Feldblum y Smith, 2005: 31-2). La movilidad de las personas altamente cualificadas es generalmente 
contemplada de forma favorable en el contexto de la liberalización del comercio internacional y se ha ido convirtiendo en un punto central de políticas nacionales e internacionales. Los efectos económicos de esta movilidad abren nuevas formas a la competencia mundial.

Las migraciones circulares conforman un caso específico en el marco de las migraciones temporales y pueden ser definidas como una forma de migración gestionada de manera tal que permite un cierto grado de movilidad legal de ida y vuelta entre dos países (Bovenkerk, 1974). La Unión Europea ha prestado atención a la migración circular stricto sensu desde 2005, probablemente introducida por primera vez en el documento de la Comisión Europea Migración y desarrollo: orientaciones concretas, de septiembre de 2005 (390) ${ }^{4}$, en el cual aparece como uno de los principales objetivos el de fomentar la transferencia de conocimientos al mundo en desarrollo evitando la "fuga de cerebros". Para ello, el fomento de la migración circular y el facilitar el retorno al país de origen constituyen las piezas clave. Así, se da prioridad de empleo a los trabajadores que ya han trabajado en regímenes temporales y han regresado al finalizar su contrato, y se facilita la migración de retorno con el fin de garantizar el éxito de la reintegración de los migrantes. Se hace un llamamiento a los Estados miembros para que compartan su experiencia en la gestión de los programas de retorno de corta duración y propone medidas como la transferencia de los derechos a pensión o el reconocimiento de las cualificaciones ${ }^{5}$. Asimismo, se propone el uso de los retornos temporales virtuales mediante, por ejemplo, la creación de sistemas de e-learning, que faciliten la creación de redes entre los investigadores extranjeros que trabajan en la UE, el apoyo a proyectos por parte de los migrantes para establecer actividades económicas sostenibles en sus países de origen y la identificación de mejores prácticas en áreas tales como la comisión de servicios y los permisos sabáticos; actuaciones que mitigan los efectos adversos de la fuga de cerebros en los países en desarrollo. Dos conceptos son especialmente relevantes: la migración circular y el retorno virtual.

- Migración circular: todo sistema gracias al cual se facilita la circulación de emigrantes o antiguos emigrantes, o la «ida y vuelta» entre el país de origen y el antiguo país de residencia.

- Retorno virtual: todo sistema que permita a cada emigrante ofrecer a su país de origen sus conocimientos o cualificaciones sin retornar físicamente a este país (es decir, principalmente gracias al recurso a las tecnologías de la información y las comunicaciones).

Otros textos han seguido a este documento tratando de desarrollar las ideas sobre las migraciones temporales y circulares, como es la comunicación a la Comisión El Planteamiento global sobre la migración un año después: Hacia una política global europea en

\footnotetext{
${ }^{4}$ Comunicación de la Comisión al Consejo, al Parlamento Europeo, al Comité Económico y Social Europeo y al Comité de las Regiones de 1 de septiembre de 2005 - Migración y desarrollo: orientaciones concretas [COM (2005) 390] Disponible en línea en: http://europa.eu/scadplus/leg/es/lvb/114166.htm (consulta el 29 de abril de 2009)

${ }^{5}$ España incorpora la nuevas propuestas de migración circular bajo el denominado "Plan de retorno voluntario". http://www.planderetornovoluntario.es/index uno.html

El plan responde al modelo de migración circular con preferencia para regresar de las personas que se acojan al plan.
} 
materia de migración, de $2006(735)^{6}$, o la comunicación, de Mayo 2007 (248), Sobre la migración circular y las asociaciones de movilidad entre la Unión Europea y terceros paises. En ésta última, y con sujeción a un examen más detallado, las medidas facilitan los procedimientos de admisión legal a las personas que ya hayan residido legalmente en la UE durante un determinado periodo de tiempo (para el desempeño de trabajo altamente cualificado, realizar estudios u otros tipos de formación). En suma, existe un claro acuerdo respecto a que las migraciones circulares suponen un nuevo enfoque jurídico en el control de las fronteras.

Las dos principales formas de migración circular que podrían ser más relevantes en el contexto de la Unión Europea, son las siguientes:

- Migración circular de nacionales de terceros países residentes en la UE: esta categoría de migración circular ofrece a las personas migrantes la oportunidad de participar en una actividad (empresarial, profesional, voluntaria o de otro tipo) en su país de origen, manteniendo al mismo tiempo su residencia principal en uno de los Estados miembros.

- Migración circular de las personas residentes en un tercer país: la migración circular podría crear una oportunidad a las personas que residen en un tercer país para llegar a la Unión Europea de forma temporal para trabajar, estudiar, formarse o una combinación de éstos, a condición de que, al finalizar el período para el que fue aprobada la entrada, regresen a su país de origen y establezcan allí su residencia y actividad principal.

Como señala la Comisión, la importancia de la migración circular reside en su utilidad como herramienta para cubrir las necesidades de mano de obra de los Estados miembros de la UE, a la vez que maximiza los beneficios de la migración para los países de origen, dado que promueve la transferencia de competencias y mitiga los riesgos de la fuga de cerebros.

Las migraciones circulares, aplicadas a todos los casos de migración en general, han sido estudiadas por el Consorcio Euro-mediterráneo para la Investigación Aplicada sobre la Migración Internacional (CARIM), que forma parte del programa AENEAS desde 2007, produciendo una serie de documentos ${ }^{8}$ sobre este tema. En esta serie aparece principalmente una revisión académica y teórica del concepto, así como la descripción de distintos casos que muestran la evolución de la cooperación bilateral entre países de la región mediterránea. No obstante, existe una notable carencia de estudios enfocados a la investigación de la migración circular, en especial la migración de personas de alta cualificación, y ello se produce a pesar de que, en la práctica, se están llevando a cabo actuaciones de esta naturaleza con creciente frecuencia.

\footnotetext{
${ }^{6}$ Comunicación de la Comisión al Consejo y al Parlamento Europeo - El Planteamiento global sobre la migración un año después: Hacia una política global europea en materia de migración [COM (2006) 735]. Disponible en línea en: http://europa.eu/scadplus/leg/es/lvb/114542.htm (consulta el 29 de abril de 2009)

7 Comunicación de la Comisión al Parlamento Europeo, al Consejo, al Comité Económico y Social y al Comité de las Regiones, de 16 de mayo de 2007, sobre la migración circular y las asociaciones de movilidad entre la Unión Europea y terceros países [COM(2007) 248]. Disponible en línea en: http://europa.eu/scadplus/leg/es/lvb/114564.htm (consulta el 29 de abril de 2009)

${ }^{8}$ Disponibles en línea en: http://www.carim.org/circularmigration
} 


\section{Migrantes de alta cualificación y la migración circular}

Como ya se ha señalado respecto a los profesionales de alta cualificación, existe una gran demanda en los países en desarrollo de este tipo de trabajadores. Huelga señalar que se trata de una demanda de difícil cobertura, al menos de forma inmediata, dada la inversión temporal (además de económica) que supone alcanzar la cualificación específica. Este hecho, distintivo del resto de las migraciones, es además matizado para los casos en los que la demanda se centra en servicios sociales básicos como la sanidad; llegándose a convertir en una demanda apremiante. Son muchos los países occidentales que lanzan, en alguna medida, llamamientos específicos orientados a profesionales de la medicina y enfermería. Estos casos suponen un claro ejemplo de migrantes de alta cualificación residentes legalmente en los países desarrollados quienes, sin embargo, no están necesariamente inscritos en el marco de la migración circular y, en ese caso, quedan al margen de los controles de coste y beneficio global propios del desarrollo de esta propuesta migratoria.

\section{Un estudio de caso: la demanda de profesionales de la salud en Europa}

Un factor clave en la prestación de la atención médica es la disponibilidad de profesionales de la salud cualificados y motivados (JLI, 2004; OMS, 2003). Sin embargo, la percepción extendida en Europa es la de que hay una inadecuada disposición y/o una mala distribución de éstos (OCDE, 2008; GTP, 2001). Algunos países del espacio europeo tienen escasez de médicos y el número de profesionales de enfermería está muy por debajo de la media de la OCDE. Si bien, respecto a estos últimos, es importante señalar las dificultades en la comparación de los datos, debido a la inclusión de diferentes categorías de enfermeros y parteros en los datos facilitados por los diferentes países. A continuación se expone un breve recorrido en cifras de las necesidades europeas relativas a profesionales sanitarios.

Finlandia contaba en 2006 con 2,7 médicos por 1000 habitantes, por debajo de la media de la OCDE que era de 3,1\%o. La cifra de enfermeros en este país, de 8,3\%o, también era inferior a la media de 9,7\% en los países de la OCDE. A raíz de una supuesta escasez de profesionales de la salud en el Reino Unido, se ha llevado a cabo una decidida y activa campaña para aumentar su número en los últimos años. Las últimas cifras muestran que en el año 2006 en el Reino Unido había 2,5\%o médicos, frente al 1,9\% de 1998, aún por debajo de la media de la OCDE de 3,1\%o, y muy por detrás de otros países europeos como Francia, Alemania, Italia y Suecia, los cuales registraban un ratio de 3,4\% o superior de médicos. Del mismo modo, se ha producido un notable incremento en el número de enfermeros en el Reino Unido en los últimos años, después de un período de relativa estabilidad. En 2006, había $11,9 \%$ enfermeros en este país en comparación con 8,0\%o en 1998. Asimismo, la media de la OCDE fue de 9,7\% enfermeros en el año 2006. También en ese año Irlanda contaba con 2,9\%o médicos, en comparación con el promedio de la OCDE de 3,1\%. Mientras que el número de médicos per cápita en Irlanda es superior a la 
de los Estados Unidos, Canadá, el Reino Unido y Australia, es menor que en muchos países europeos. Por otro lado, Irlanda tiene un número muy elevado de practicantes de enfermería, con 15,4\% enfermeros en 2006, significativamente por encima de la media de la OCDE de 9,7\% . En ese mismo año en Polonia había 2,2\% médicos, frente al promedio de 3,1\% de todos los países de la OCDE; además de un ratio de enfermeros cualificados de 5,1\%o, también por debajo de la media en los países de la OCDE de 9,7\%o. A pesar del nivel relativamente bajo de gasto en salud en Italia, hay más médicos per cápita que en la mayoría de otros países de la OCDE. En 2006 en Italia había 3,7\% de practicantes de medicina, por encima de la media de la OCDE de 3,1\%o. Por otra parte, había en ese mismo año en el país 7,1\%o enfermeros, una cifra inferior a la media de 9,7\%o en los países de la OCDE. En 2005, en Portugal había 3,4\%o médicos, ligeramente por encima de la media de la OCDE (3,1\%o médicos). Aunque el número de enfermeros en Portugal por 1000 habitantes ha aumentado en los últimos años, de 2,8\%o enfermeros en 1990 a 4,6 en 2005, Portugal está aún por detrás de la media de la OCDE de 9,7\%o enfermeros. En 2004, la República Eslovaca, había 3,1\%o médicos, que es también la media de los 30 países de la OCDE, y 6,3\%o enfermeros en 2004, por debajo de la media en los países de la OCDE de 9,7\% . Hungría contaba en 2006 con 3,0\%o médicos, similar a la media de la OCDE de 3,1\%o, y 6,1\%o enfermeros cualificados, por debajo de la media en los países de la OCDE de 9,7\%o. En cuanto a Grecia, es el país de la OCDE donde más médicos per cápita hay; durante las últimas décadas, el número de médicos per cápita aumentó rápidamente en Grecia para llegar a 5\%o médicos en 2005, muy por encima de la media de la OCDE de 3,1\%. Por otro lado, sólo había 3,3\% enfermeros en este país en 2005 , una cifra mucho más baja que la media de 9,7\% en los países de la OCDE.

Para el caso de España, hay más médicos per cápita que en la mayoría de los países de la OCDE. En 2006, España había 3,6\% médicos, por encima de la media de la OCDE de 3,1\%o. Pero, por otro lado, se contaba con una proporción de 7,3\%0 enfermeros cualificados, una cifra inferior a la media de $9,7 \%$ en los países de la OCDE en ese año.

Estas cifras muestran los desequilibrios en el espacio europeo en general y en muchos países de éste en particular, respecto a la oferta y la demanda de profesionales sanitarios. La preocupación por este tema ha impulsado estudios referidos a las características concretas tanto de la oferta como de la demanda en este ámbito, contando desde la UE (2008) como texto referente a este respecto con Green Paper. On the European Workforce for Health, (725/3)9: la migración circular aparece en éste como una herramienta de acción. En nuestro país, el estudio Ofertay necesidad de especialistas médicos en España (2008-2025)10 aborda el papel de la migración en este tema, no obstante, sin llegar a mencionar la migración circular como un programa orientado a paliar los desajustes y capitalizar los recursos humanos.

\footnotetext{
9 Green Paper. On the European Workforce for Health. Brussels COM (2008) 725/3. Disponible en línea en: http://ec.europa.eu/health/ph systems/docs/workforce gp en.pdf

${ }^{10}$ Disponible en línea en: http://www.msps.es/profesionales/formacion/docs/necesidadesEspecialistas2008_2025.pdf
} 


\section{Perfil de los migrantes de alta cualificación en el área de la salud}

La carencia general de profesionales de la salud en estos países ha sido cubierta por migrantes de alta cualificación procedentes de terceros países. En cuanto a la procedencia específica de éstos, resulta difícil perfilar un único modelo, existiendo distintos perfiles de médicos extranjeros en diversos países de la Unión Europea. Así, mientras que en países como Austria, Noruega, Portugal y Francia, la mitad del total de médicos extranjeros no provienen de otros países de la Unión Europea, las tres cuartas partes de los médicos extranjeros en el Reino Unido y, posiblemente, en Irlanda, proceden de países no pertenecientes a la UE y países en desarrollo en general, una situación que puede dar lugar a cuestiones éticas (Simoens y Hurst, 2004; Eastwood et al., 2005). En suma, el origen de estos profesionales de la salud es una cuestión clave en la evaluación del impacto que puede tener la migración profesional en diferentes países (Jakubowski, 2004, Nicholas, 2004). 


\section{Referencias Bibliográficas}

Bovenkerk, Frank (1974) The sociology of return migration: a bibliograpic essay. La Haya, Martinus Nijhoff

Pierre Casarino (ed) (2008): "Conditions of Modern Return Migrants", International Journal on Multicultural Societies, Vol. 10, No. 2, UNESCO.

Dumont JC, Zurn P (2007): "Immigrant health workers in OECD countries in the broader context of highly skilled migration". En Organisation for Economic Co-operation and Development OECD. International Migration Outlook. Annual Report, 2007 Edition. OECD Publishing;

Eastwood JB, Conroy RE, Naicker S, West PA, Tutt RC, Plange-Rhule J: "Loss of health professionals from sub-Saharan Africa: the pivotal role of the UK". Lancet 2005, 365(9474):1893-1900.

Fargues, P. "Circular Migration: Is it relevant for the South and East of the mediterranean?" CARIM. Analytical and Synthetic Notes 2008/40. Circular Migration Series. www.carim.org

Favell, A., Feldblum M., M. P. Smith (2006) 'The human face of global mobility: A research agenda' in Michael Peter Smith Adrian Favell eds., The Human Face of Global

González López B. y Barber P. (2009) Oferta y necesidad de médicos especialistas en España (2006-2030). Universidad de las Palmas de Gran Canaria/Ministerio de Sanidad y Consumo. Disponible en línea en:

http://www.msps.es/profesionales/formacion/docs/necesidadesEspecialist as2008 2025.pdf

Mobility: International Highly Skilled Migration in Europe, North America and the AsiaPacific. Comparative Urban and Community Research, vol.8. (New Brunswick, NJ: Transaction Press).

Jakubowski E, Hess R (2004) "The market for physicians". En Health policy and European Union enlargement. McKee M, MacLehose L, Nolte E. Berkshire (Eds.) (England): Open University Press; pp. 131-142.

Joint Learning Initiative (2004) Human resources for health. Overcoming the crisis. Cambridge (Mass., US): Harvard University Press.

http://www.healthgap.org/camp/hcw docs/JLI exec summary.pdf

Mullan F: The metrics of the physician brain drain. N Engl J Med 2005, 353(17):1810-8 
Nicholas S (2004) "The challenges of the free movement of health professionals". En Health policy and European Union enlargement. McKee M, MacLehose L, Nolte E. Berkshire (Eds.) (England): Open University Press; 2004, 82-108.

Simoens S, Hurst J: "Matching supply with demand for the services of physicians and nurses". En The OECD Health Project. Towards high performing health systems. Policy studies. Paris: OECD Publications; 2004.

Permanent Working Group of European Junior Doctors (PWG): Medical manpower in Europe by the year 2000: from surplus to deficit? In Handbook of Policy Statements. An update of the 1997 handbook. Oporto: PWG; 2001:38-41.

Sabour, M'hammed (2001) The Ontology and Status of Intellectuals in Arab Academia and Society. Burlington, England: Ashgate Publishing).

OECD Health Data 2008: Statistics and Indicators for 30 Countries

World Health Organization (2003) Human resources and national health systems. Shaping the agenda for action. Geneve: World Health Organization http://www.who.int/entity/hrh/documents/en/nhs shaping agenda.pdf 